\title{
Solitary fibrous tumors of the pleura with Doege-Potter syndrome: a case report and three-decade review of the literature
}

Wen Meng ${ }^{1 \dagger}$, Hong-Hong Zhu ${ }^{2 *}{ }^{*}, \mathrm{Hu} \mathrm{Li}^{1}$, Guoqing Wang ${ }^{1}$, Dongshan Wei ${ }^{1}$ and Xing Feng ${ }^{1}$

\begin{abstract}
Background: No case of solitary fibrous tumor of the pleura with Doege-Potter syndrome has been reported in China. This study was to report a rare repeatedly recurrent case of solitary fibrous tumor of the pleura with Doege-Potter syndrome diagnosed in China and a three-decade literature review of solitary fibrous tumor of the pleura with Doege-Potter syndrome worldwide.

Case presentation: A rare case of solitary fibrous tumor of the pleura with Doege-Potter syndrome was diagnosed in 2005 with follow-up to 2011. All medical records were collected and literature of solitary fibrous tumor of the pleura with Doege-Potter syndrome from 1979 to 2011 was obtained through Medline. This typical case, diagnosed and confirmed by histopathologic results, was a 72-year-old Chinese woman who had a complaint of night sweat for a month. A localized mass $12 \mathrm{~cm} \times 11 \mathrm{~cm} \times 8 \mathrm{~cm}$ in size was found associated with pleural effusion in her left low chest cavity, and blood tests showed severe hypoglycemia. Removal of the mass solved the hypoglycemia. The case was repeatedly recurrent in April, 2010 and March, 2011 and had no signs of recurrence up to the end of 2011 after surgery. A review of 45 cases of solitary fibrous tumor of the pleura with Doege-Potter syndrome compared and summarized clinical characteristics, treatments, and outcomes by benign and malignant tumor nature.

Conclusions: Incidence of solitary fibrous tumor of the pleura with Doege-Potter syndrome is similar between genders. There are no significant differences in clinical characteristics between benign and malignant cases. Surgery is the first effective treatment for solitary fibrous tumor of the pleura with Doege-Potter syndrome and the completeness of the initial resection is the key to preventing recurrence. Routine follow-up examinations are recommended for early detection of recurrence.
\end{abstract}

Keywords: Solitary fibrous tumors of pleura, Doege-Potter syndrome, Surgery, Case report review

\section{Background}

Solitary fibrous tumor of the pleura (SFTP) is a rare primary tumor, occupying less than $5 \%$ of all pleural tumors [1]. It arises from the submesothelial mesenchymal layer. Hypoglycemia accompanying SFTP is specifically referred to as the Doege-Potter syndrome (DPS) [2]. A large form of insulin-like growth factor 2 (IGF-2), which is probably an incompletely processed molecule of IGF-2, is derived from the tumor and considered to be a hypoglycemic mechanism [3]. England reported pathologic

\footnotetext{
* Correspondence: hozhu@jhsph.edu

†Equal contributors

${ }^{2}$ Department of Public Health, College of Health and Human Service,

Western Kentucky University, Bowling Green, KY 42101, USA

Full list of author information is available at the end of the article
}

differences between benign and malignant SFTP among 141 benign and 82 malignant neoplasms [4] but no summary of clinical characteristics of benign and malignant SFTP with DPS due to a small number $(n=12)$ of DPS cases. Our report presented a typical case of repeatedly recurrent malignant SFTP with DPS in China, followed from 2005 to 2011. We then reviewed the literature of case reports of SFTP with DPS from 1979 to 2011 through Medline, and compared and summarized clinical characteristics, treatments, and outcomes by benign and malignant nature.

\section{Case presentation}

A 72-year-old Chinese woman was hospitalized with a complaint of night sweat for one month in January, 2005.

\section{Biomed Central}


A localized mass $12 \mathrm{~cm} \times 11 \mathrm{~cm} \mathrm{x} 8 \mathrm{~cm}$ in size was found associated with pleural effusion in her left low chest cavity. Blood tests showed she was severely hypoglycemic. Removal of the mass solved the hypoglycemia. No other treatments were given. The histopathologic result showed a solitary fibrous tumor, potentially malignant.

The patient was followed up until April, 2010 when symptoms of night sweats returned. A physical examination showed she had no breath sound in the left low cavity, and the percussion note was dull on the left side of her thorax. Laboratory evaluation revealed no significant abnormalities in hematological or biochemical profile except low blood glucose. The complete resection of the mass revealed multiple well-capsulated masses in the left low chest cavity, the largest one $9 \mathrm{~cm} \times 5 \mathrm{~cm} \times 4 \mathrm{~cm}$ in size. Local tumor necrosis and mitosis up to 4/10 high power fields were microscopically identified. In March, 2011, the patient again had symptoms of night and morning sweats, and computed tomography and X-ray revealed a large recurrent tumor mass. The extensive excision of mass plus extensive excision of the pleura was performed revealing multiple recurrent parietal pleural masses in the left low chest cavity, the largest measured $14 \mathrm{~cm} \times 10 \mathrm{~cm} \times 10 \mathrm{~cm}$. The episodes of hypoglycemia abated postoperatively and the patient seemed in good health after the surgery and had no signs of recurrence when we followed her up to the end of 2011.

\section{Literature review}

We reviewed the literature in both English and Chinese languages from 1979 to 2011 through Medline and summarized 45 cases of SFTP with DPS and classified them into 22 benign and 23 malignant cases based on histopathologic results, referring to the criteria by England et al. [4].

\section{Summary of the 45 cases of SFTP with DPS Basic characteristics}

The age at diagnosis among 22 benign patients ranges from 38 to 79 years old, with a mean age of $60.8 \pm 10.0$ (standard deviation) and 23 malignant patients 31 to 79 years with a mean age of $64.1 \pm 10.8$. Twenty-four cases (12 benign and 12 malignant) are females and 21 (10 benign and 11 malignant) males. About $86.4 \%$ of benign and $87.0 \%$ of malignant SFTP patients are diagnosed at the ages of 50-70 years old (Table 1 ).

\section{Clinical characteristics}

All patients have hypoglycemia-related symptoms. Abnormal consciousness, confusion, and sweating are often seen in both benign and malignant patients. Signs (such as pleural effusion, clubbing in fingers, and skin change) are also seen in both benign and malignant patients (Table 1 ).

From the 13 benign and 20 malignant reports that have a detailed description of the tumor mass, $100.0 \%$
Table 1 Summary characteristics of $\mathbf{4 5}$ cases of solitary fibrous tumors of the pleura with Doege-Potter syndrome, 1979-2011

\begin{tabular}{|c|c|c|c|}
\hline Characteristic & $\begin{array}{l}\text { Benign } \\
(n=22)\end{array}$ & $\begin{array}{l}\text { Malignant } \\
(n=23)\end{array}$ & $P$-value* \\
\hline Age at diagnosis (years): Mean $\pm \mathrm{SD}^{\lessgtr}$ & $60.8 \pm 10.0$ & $64.1 \pm 10.8$ & 0.29 \\
\hline \multicolumn{4}{|l|}{ Gender } \\
\hline Male & 10 & 11 & 0.87 \\
\hline Female & 12 & 12 & \\
\hline \multicolumn{4}{|l|}{ Sign } \\
\hline Pleural effusion & $5(n=16)$ & $4(n=16)$ & 0.69 \\
\hline Clubbing in fingers & $5(n=16)$ & $4(n=16)$ & 0.69 \\
\hline Skin change & $2(n=16)$ & $1(n=16)$ & 0.54 \\
\hline \multicolumn{4}{|l|}{ Tumor mass } \\
\hline Clear borders & $13(n=13)$ & $16(n=20)$ & 0.09 \\
\hline \multicolumn{4}{|l|}{ Location } \\
\hline Right & $13(n=21)$ & $14(n=23)$ & 0.94 \\
\hline Left & $8(n=21)$ & $9(n=23)$ & 0.94 \\
\hline Up & $1(n=17)$ & $2(n=20)$ & 0.65 \\
\hline Low & $16(n=17)$ & $18(n=20)$ & 0.65 \\
\hline \multicolumn{4}{|l|}{ Size $(\mathrm{cm})$} \\
\hline$\leq 10$ & $0(n=17)$ & $1(n=21)$ & \\
\hline$>10$ & $17(n=17)$ & $20(n=21)$ & 0.36 \\
\hline \multicolumn{4}{|l|}{ Morphologic Features } \\
\hline Homogeneous & $2(n=5)$ & $3(n=17)$ & \\
\hline Heterogeneous & $3(n=5)$ & $14(n=17)$ & 0.29 \\
\hline
\end{tabular}

*Pearson $P$-values for all frequency variables were estimated by individual Chi-square test and for age at diagnosis by $t$-test.



benign and $80.0 \%$ malignant have sharply circumscribed borders. Most tumors are located in the right low area of chest cavity. The size of most benign and malignant tumors is greater than $10 \mathrm{~cm}$. The average dimension of these SFTPs with DPS is $21.7 \pm 5.0 \mathrm{~cm}$ for benign and $17.6 \pm 4.1 \mathrm{~cm}$ for malignant tumors. There is no significant difference in morphologic features between benign and malignant tumors (Table 1).

\section{Treatments and outcomes}

Of the 18 benign cases of SFTP with DPS with detailed surgical information, $13(72.2 \%)$ are removed by simple excision of mass, and five cases by complex excision that included adhesion tissue. Of the 20 malignant cases with detailed surgical information, 11 (55.0\%) are removed by simple excision, and $9(45.0 \%)$ cases by complex excision. No distant and lymph node metastasis are found in all cases. The follow-up time in 29 (64.4\%) of 45 cases ranges from 1 to 288 months (average time, $35.3 \pm 57.2$ months). All but four (two in benign and two in malignant) of the patients are free of recurrent SFTP with DPS. One 
recurrent tumor is performed by complex excision (recurrent tumor and adherent tissue). One recurrent tumor is performed by recurrent tumor excision. But they all are experienced repeated relapses. The other two patients refuse medical advice based on poor lung function and other reasons. Radiotherapy and chemotherapy are used in four malignant cases - two with single radiotherapy and chemotherapy but none of them show any effect or benefit. Two of three cases having detailed information of positron emission tomography-computed tomography examination show little to moderate uptake of $\left[{ }^{14} \mathrm{C}\right]$ fluorodeoxyglucose. Five of 20 cases given transthoracic needle biopsy could not get diagnosed, and seven of 20 cases could not be differentiated between benign and malignant SFTP due to limitation of tissue (Table 2).

\section{Discussion}

Some criteria, especially England [4], are used worldwide to distinguish benign from malignant SFTP [5]. The criteria defined by England et al. [4] include high cellularity, pleomorphism with cytonuclear atypia, more than

\begin{tabular}{|c|c|c|c|}
\hline Treatment/outcome & $\begin{array}{l}\text { Benign } \\
(n=22)\end{array}$ & $\begin{array}{l}\text { Malignant } \\
(\mathrm{n}=23)\end{array}$ & $P$-value* \\
\hline
\end{tabular}

Treatment

Surgery

Simple excision

$13(n=18) \quad 11(n=20)$

Complex excision

$5(n=18) \quad 9(n=20) \quad 0.27$

Radiotherapy

Chemotherapy

$0 \quad 1(n=23)$

Combination therapy

Surgery + radiotherapy

Surgery + Chemotherapy

Surgery + radio + Chemotherapy

Recurrent

Imaging features

Lobular borders

Smooth borders

Pedicle

Calcification

Mass effect on mediastinum

Atelectasis

Chest wall involvement

Results of PET $C T^{\$}$

0

$1(n=23)$

$\begin{array}{ll}0 & 2(n=23) \\ 0 & 0 \\ 0 & 0 \\ 2(n=14) & 2(n=15) \quad 0.94\end{array}$

$6(n=11) \quad 9(n=15)$

$5(n=11) \quad 6(n=15) \quad 0.78$

$3(n=11) \quad 5(n=16) \quad 0.82$

$2(n=9) \quad 0$

$8(n=9) \quad 8(n=13) \quad 0.16$

$8(n=9) \quad 7(n=9) \quad 0.53$

$1(n=12) \quad 3(n=8) \quad 0.11$

$2(n=3) \quad 0$

Benefit of transthoracic needle biopsy

$8(n=11) \quad 7(n=9)$

0.80

*Pearson $P$-values for all frequency variables were estimated by individual Chi-square test.

${ }^{\$}$ PET CT: positron emission tomography-computed tomography. four mitoses per ten high-power fields, associated necrotic or hemorrhagic areas. At least $80 \%$ of all SFTPs are benign, while the rest may show characteristics of malignant tumor $[2,4,6]$. From our study, there are roughly equal numbers of cases between benign and malignant SFTP with DPS which is higher than $12-13 \%$ of malignancy reported in literature [6]. Gender is nearly evenly distributed between the benign (12 women and 10 men) and malignant (12 women and 11 men), which is different from the results reported by England that DPS was three times as frequent in females as in males [4], probably due to the small number of DPS cases in his study. Most SFTPs associated with DPS are located in the right lower hemithorax, which is similar to England's results [4]. The sizes of 100\% benign and 95.2\% malignant tumors are $>10 \mathrm{~cm}$, similar to the conclusion from previous studies $[2,4]$.

Based on the information of 45 cases of SFTP with DPS in our study, the clinical performances between benign and malignant tumors are almost the same. Local recurrence can occur in both benign and malignant tumors of SFTP with DPS (two of 14 benign and two of 15 malignant). Surgery is the first effective treatment for both the original and recurrent benign and malignant masses [7-9]. These findings are also confirmed by our case report. Also, we think the completeness of initial resection is the key to preventing recurrence of SFTP with DPS. Some recurrent patients may lose the chance to get re-excision due to unfavorable health condition after the surgery. For the tumors located in parietal pleura, complete resection plus extensive excision of the pleura should be adopted in order to prevent recurrence. It is noteworthy that four cases (three malignant, one benign) performed transformation from non-hypoglycemia to hypoglycemia and one recurrent case performed malignant transformation 25 years after the surgery [10]. Routine follow-up examinations are recommended for early detection of recurrent SFTP. Our study also supports the finding that neither radiotherapy nor chemotherapy has any effect on the treatment of SFTP with DPS.

\section{Conclusions}

Incidence of SFTP with DPS is similar between genders. There are no significant differences in clinical characteristics between benign and malignant cases. Surgery is the first effective treatment for SFTP with DPS and the completeness of the initial resection is the key to preventing recurrence. Routine follow-up examinations are recommended for early detection of recurrent SFTP with DPS.

\section{Consent}

Written informed consent was obtained from the patient for publication of this Case Report and any accompanying images. A copy of the written consent is available for review by the Editor-in-Chief of this journal. 


\section{Abbreviations}

SFTP: Solitary fibrous tumors of pleura; DPS: Doege-Potter syndrome; IGF-2: Insulin-like growth factor 2.

\section{Competing interests}

Authors have no financial and non-financial competing interests with anyone or any individual organizations.

\section{Authors' contributions}

WM conducted part of case reports collection, management and analysis, and drafted the manuscript. HHZ did data correction, conducted part of case reports collection, did data analysis and interpretation, and co-drafted and revised the manuscript. HL conducted diagnosis and treatment of patient. GQW performed operation work on patient. DSW and XF did follow-up work and provided a detailed description of the patient. All authors read and approved the final manuscript.

\section{Acknowledgements}

The authors thank physicians, nurses and staff in the Department of Cardiothoracic Surgery, Hangzhou First People's Hospital, for their assistance in the case diagnosis and treatment.

\section{Author details}

'Department of Cardiothoracic Surgery, Hangzhou First People's Hospital, Hangzhou 310006, Zhejiang, Province P R China. ${ }^{2}$ Department of Public Health, College of Health and Human Service, Western Kentucky University, Bowling Green, KY 42101, USA.

Received: 16 September 2013 Accepted: 18 July 2014

Published: 11 August 2014

\section{References}

1. Furukawa N, Hansky B, Niedermeyer J, Gummert J, Renner A: A silent gigantic solitary fibrous tumor of the pleura: case report. J Cardiothorac Surg 2011, 6:122

2. Kalebi AY, Hale MJ, Wong ML, Hoffman T, Murray J: Surgically cured hypoglycemia secondary to pleural solitary fibrous tumour: case report and update review on the Doege-Potter syndrome. J Cardiothorac Surg 2009, 4:45.

3. Daughaday WH, Kapadia M: Significance of abnormal serum binding of insulin-like growth factor II in the development of hypoglycemia in patients with non-islet-cell tumors. Proc Natl Acad Sci U S A 1989, 86:6778-82.

4. England DM, Hochholzer L, McCarthy MJ: Localized benign and malignant fibrous tumors of the pleura. A clinicopathologic review of 223 cases. Am J Surg Pathol 1989, 13:640-658.

5. Travis WD, Brambilla E, Muller-Hermelin HK, Harriset CC: World Health Organization Classification of Tumours. Pathology and Genetics of Tumours of the Lung, Pleura, thymus and Heart. Lyon: IARC Press; 2004:142-143.

6. Zafar $\mathrm{H}$, Takimoto $\mathrm{CH}$, Weiss $\mathrm{G}$ : Doege-Potter syndrome: hypoglycemia associated with malignant solitary fibrous tumor. Med Oncol 2003, 20:403-8.

7. Gold JS, Antonescu CR, Hajdu C, Ferrone CR, Hussain M, Lewis JJ, Brennan MF, Coit DG: Clinicopathologic correlates of solitary fibrous tumors. Cancer 2002, 94:1057-68

8. Santos RS, Haddad R, Lima CE, Liu YL, Misztal M, Ferreira T, Boasquevisque CH, Luketich JD, Landreneau R: Patterns of recurrence and long-term survival after curative resection of localized fibrous tumors of the pleura. Clin Lung Cancer 2005, 7:197-201.

9. Robinson LA: Solitary fibrous tumor of the pleura. Cancer Cause 2006, 13:264-9.

10. Mohamed H, Mandal AK: Natural history of multifocal solitary fibrous tumors of the pleura: a 25-year follow-up report. J Natl Med Assoc 2004, 96:659-64.

doi:10.1186/1756-0500-7-515

Cite this article as: Meng et al: Solitary fibrous tumors of the pleura with Doege-Potter syndrome: a case report and three-decade review of the literature. BMC Research Notes 2014 7:515.

\section{Submit your next manuscript to BioMed Central and take full advantage of:}

- Convenient online submission

- Thorough peer review

- No space constraints or color figure charges

- Immediate publication on acceptance

- Inclusion in PubMed, CAS, Scopus and Google Scholar

- Research which is freely available for redistribution 